\title{
Plasma emission spectroscopy of solids irradiated by intense XUV pulses from a free electron laser
}

\author{
T.W.J. Dzelzainis a ${ }^{\text {, J. Chalupsky }}{ }^{\mathrm{b}}$, M. Fajardo ${ }^{\mathrm{c}}$, R. Fäustlin ${ }^{\mathrm{d}}$, P.A. Heimann ${ }^{\mathrm{k}}$, V. Hajkova ${ }^{\mathrm{b}}$, L. Juha ${ }^{\mathrm{b}}$, \\ M. Jurek ${ }^{b}$, F.Y. Khattak ${ }^{f}$, M. Kozlova ${ }^{b}$, J. Krzywinski ${ }^{\text {h }}$, R.W. Lee ${ }^{e}$, B. Nagler ${ }^{i}$, A.J. Nelson ${ }^{\text {e }}$, F.B. Rosmej ${ }^{j}$, \\ R. Soberierski ${ }^{g}$, S. Toleikis ${ }^{d}$, T. Tschentscher ${ }^{d}$, S.M. Vinko ${ }^{i}$, J.S. Wark ${ }^{i}$, T. Whitcher ${ }^{i}$, D. Riley ${ }^{\text {a,* }}$ \\ a School of Mathematics and Physics, Queen's University, Belfast, Belfast BT7 1NN, UK \\ ${ }^{\mathrm{b}}$ Institute of Physics, AS CR, Prague 8, Czech Republic \\ ${ }^{\mathrm{C}}$ Centro de Fisica dos Plasmas, Instituto Superior Técnico, Lisboa, Portugal \\ ${ }^{\mathrm{d}}$ Deutsches Elektron-Synchrotron DESY, Notkestrasse 85, 22607 Hamburg, Germany \\ e Lawrence Livermore National Laboratory, University of California, P.O. Box 808, Livermore, CA 94551, USA \\ ${ }^{\mathrm{f}}$ Department of Physics, Kohat University of Science and Technology, Kohat-26000, NWFP, Pakistan \\ $\mathrm{g}$ Inst. of Physics, Polish Academy of Sciences, Poland \\ ${ }^{\text {h }}$ SLAC National Accelerator Laboratory 2575 Sand Hill Road, Menlo Park, CA 94025 USA \\ ${ }^{\mathrm{i}}$ Clarendon Laboratory, University of Oxford, South Parks Road OX1 3PU, UK \\ ${ }^{j}$ Universite Pierre et Marie Curie, UMR 7605 case 128, 4 Place Jussieu, 75252 Paris Cedex 05, France \\ ${ }^{\mathrm{k}}$ Lawrence Berkeley National Laboratory, 1 Cyclotron Road Berkeley, CA 94720 USA
}

\section{A R T I C L E I N F O}

\section{Article history:}

Received 22 May 2009

Accepted 22 May 2009

Available online 30 May 2009

\section{Keywords:}

XUV spectroscopy

VUV-FEL

Warm dense plasma

\begin{abstract}
A B S T R A C T
The FLASH XUV-free electron laser has been used to irradiate solid samples at intensities of the order $10^{16} \mathrm{~W} \mathrm{~cm}^{-2}$ at a wavelength of $13.5 \mathrm{~nm}$. The subsequent time integrated XUV emission was observed with a grating spectrometer. The electron temperature inferred from plasma line ratios was in the range $5-8 \mathrm{eV}$ with electron density in the range $10^{21}-10^{22} \mathrm{~cm}^{-3}$. These results are consistent with the saturation of absorption through bleaching of the L-edge by intense photo-absorption reported in an earlier publication.
\end{abstract}

(C) 2009 Elsevier B.V. All rights reserved.

\section{Introduction}

The study of warm dense matter is of intense interest to many researchers in the fields of plasma physics, fusion sciences, planetary and astrophysical sciences. The reasons for this include the fact that, theoretically, such matter is difficult to handle, with electrostatic and thermal energies on a par with each other, rather than one being a perturbation of the other as in solid state physics or classical plasma physics. Experimental data is not easy to obtain and one of the key restraints is the ability to create uniform well defined samples of matter at around solid density but with temperatures of $\sim 1-100 \mathrm{eV}$. A possible way forward is the use of the new generation of XUV-free electron lasers (XUV-FEL) to irradiate solid samples [1-4]. These devices do not suffer from the critical density problem associated with short pulse optical laser irradiation, where the energy is absorbed at critical electron

\footnotetext{
* Corresponding author. Tel./fax: +44 2890973500.

E-mail address: d.riley@qub.ac.uk (D. Riley).
}

density (of order $10^{21}-10^{22} \mathrm{~cm}^{-3}$ ) rather than directly at the solid density. Furthermore, intense XUV irradiation of solids is of great interest for those investigating future lithography techniques. In this paper we report an experiment in which solid foils (Al, Fe and Co) were irradiated with intense pulses of $13.5 \mathrm{~nm}$ radiation with the FLASH XUV-FEL. The pulses were of $15 \mathrm{fs}$ duration and focussed to $2 \mu \mathrm{m}$ with a multi-layer optic to achieve intensities of around $10^{16} \mathrm{~W} \mathrm{~cm}^{-2}$

\section{Experimental set-up}

The experimental arrangement is shown in schematic form in Fig. 1. The XUV beam at $13.5 \mathrm{~nm}$ was running at $5 \mathrm{~Hz}$. A $3 \mathrm{~mm}$ aperture in the beam limited the size of the beam and the pulse energies were typically 5-10 $\mu \mathrm{J}$ on target. The beam was focussed with a multi-layer Mo-Si optic with a focal length of $269 \mathrm{~mm}$ [5]. The best focus was established by analysis of PMMA ablation using a range of target positions relative to the focus optic and a range of pulse energies. This analysis, which used a Normarski differential 


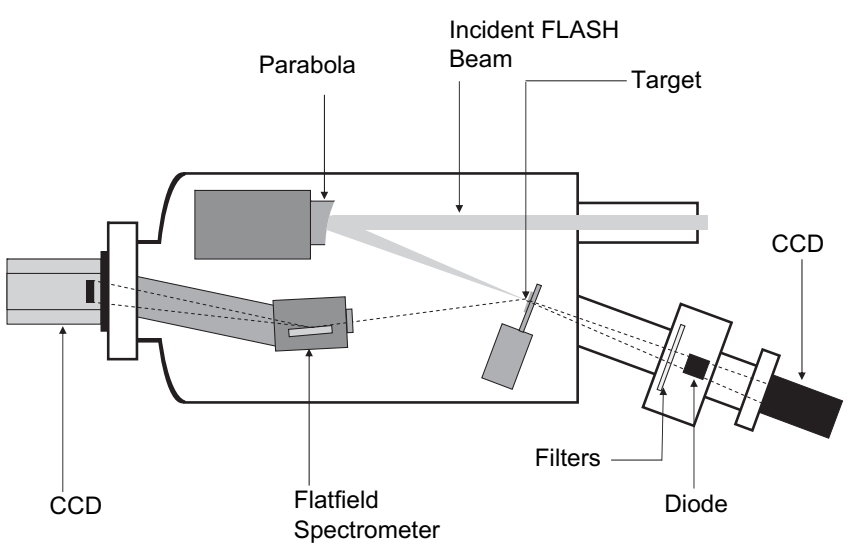

Fig. 1. Schematic of the experimental layout

interference microscope has established that the best focus was $\sim 2.3 \mu \mathrm{m}$ FWHM, giving a fluence on target of $160 \mathrm{~J} \mathrm{~cm}^{-2}$ with intensity of $\sim 10^{16} \mathrm{~W} \mathrm{~cm}^{-2}$. The reflectivity of the optic was measured after the experiment and found to be $48 \%$. A grating spectrometer, using an Hitachi, $1200 \mathrm{l} / \mathrm{mm}$ variable line spacing grating was used to create a flat spectral focus plane on a CCD camera positioned on a vacuum flange with the chip in vacuum.

The spectral range covered was from 10 to $30 \mathrm{~nm}$ and the second and third harmonics efficiencies of this type of grating have been investigated previously [6]. An Al edge filter was used to establish that the spectral resolution achieved was $0.1 \mathrm{~nm}$. A Questar QM1 long distance microscope coupled to a high quality mirror was used to image the front of the target surface during the experiment. The damage spots created by the incident FEL beam could be readily observed to check that during a shot series a fresh surface was exposed for each shot. This telescope was also used to establish, prior to the experimental runs, that the target surface remained within a few microns of the pre-set focal position as the target was rastered a distance of $20 \mathrm{~mm}$ across the focus - thus focussing conditions were consistently well within the Rayleigh depth for a particular run. An Al foil target $10 \mu \mathrm{m}$ thick was moved across the focus continuously, to expose a fresh surface to each shot. The position of the target relative to the focus was changed between best focus and $1.3 \mathrm{~mm}$ away from best focus, thus changing the focal spot from $\sim 2.3 \mu \mathrm{m}$ to $\sim 16 \mu \mathrm{m}$. In Fig. 2 we can see some sample spectra integrated over many shots.

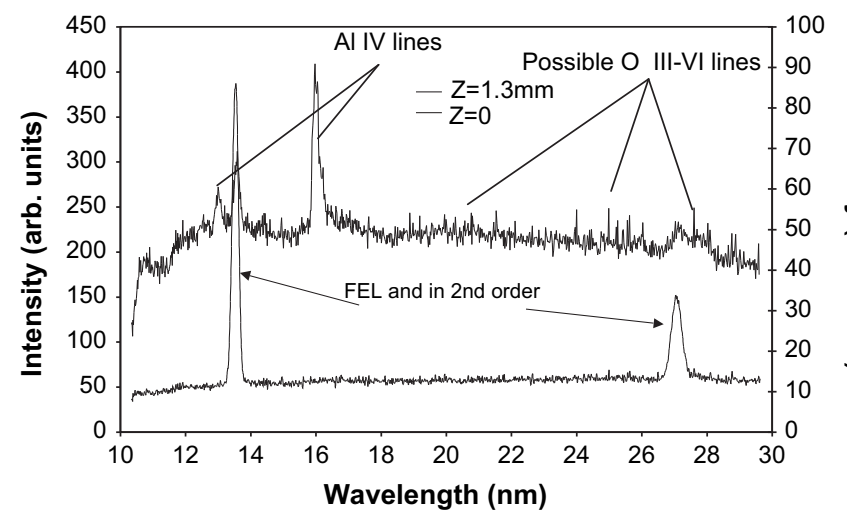

Fig. 2. Upper curve: Emission spectra taken at best focus, $Z=0$ integrated over 3000 shots and lower curve: far from focus integrated over 1000 shots. The intensity is scaled to account for the different number of integrations.
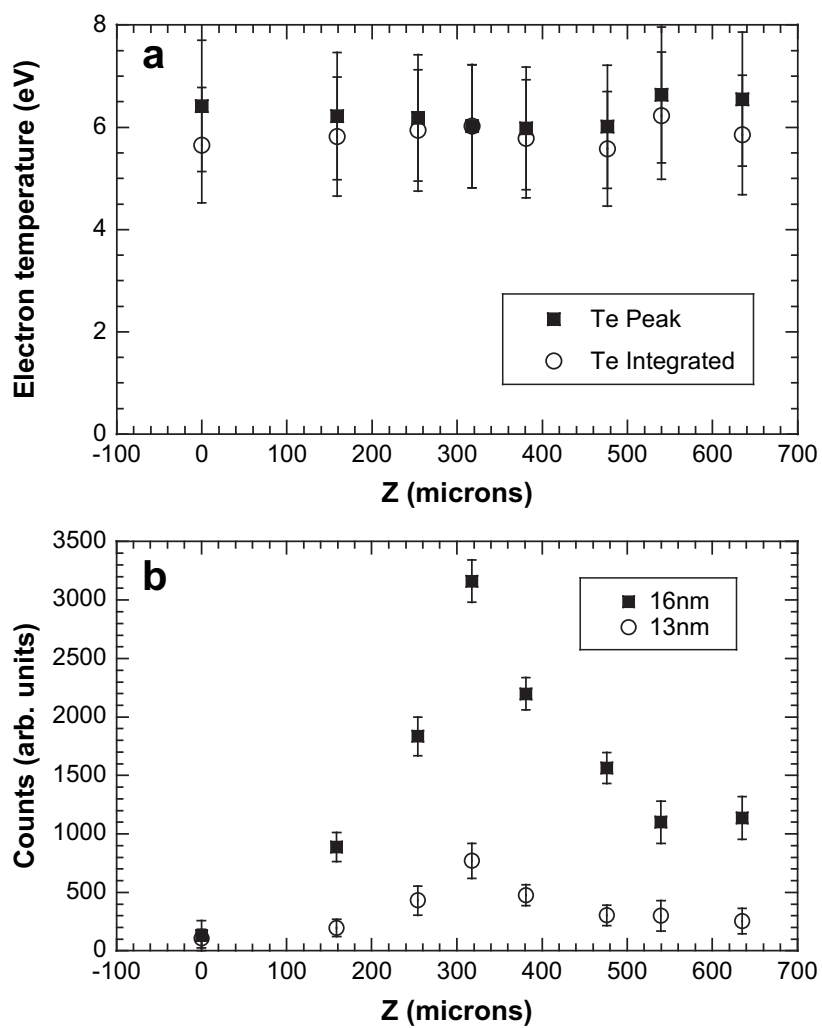

Fig. 3. (a) Estimated electron temperature based on line ratio from Al IV lines using both peak of the lines and frequency integrated intensity; (b) Variation in emission of Al IV lines as a function of focal plane position.

We can see that in the low intensity case $\left(\mathrm{I} \sim 1.7 \times 10^{14} \mathrm{~W} \mathrm{~cm}^{-2}\right)$ we observe only the scattered FEL light in first and second order. In the tight focus case $\left(\mathrm{I} \sim 8 \times 10^{15} \mathrm{~W} \mathrm{~cm}^{-2}\right.$ ) we can see strong additional features at $\sim 13 \mathrm{~nm}$ and $\sim 16 \mathrm{~nm}$ identified [7-9] as the Al IV lines; $2 \mathrm{~s}^{2} 2 \mathrm{p}^{6}-2 \mathrm{~s}^{2} 2 \mathrm{p}^{5}\left({ }^{2} \mathrm{P}^{0}\right) 3 \mathrm{~d}{ }^{1} \mathrm{P}$ and ${ }^{3} \mathrm{D}$ lines at $12.97 \mathrm{~nm}$ and $13.04 \mathrm{~nm}$ respectively and the $2 \mathrm{~s}^{2} 2 \mathrm{p}^{6}-2 \mathrm{~s}^{2} 2 \mathrm{p}^{5}\left({ }^{2} \mathrm{P}^{0}\right) 3 \mathrm{~s}^{1} \mathrm{P}$ and ${ }^{3} \mathrm{P}$ lines at $16.01 \mathrm{~nm}$ and $16.17 \mathrm{~nm}$ respectively. In addition, there are weaker features tentatively identified as emission from hollow $\mathrm{Al}$ ions that may originate from the solid density phase and will be the subject of a subsequent paper. The fall-off in signal below $12 \mathrm{~nm}$ is due to the response of the grating [6]. The feature to the right of the 2nd order FEL may come from $\mathrm{O}$ emission due to the several $\mathrm{nm}$ thick layer of oxide generally present on Al. There is also some evidence (although not conclusive) of a large number of weaker

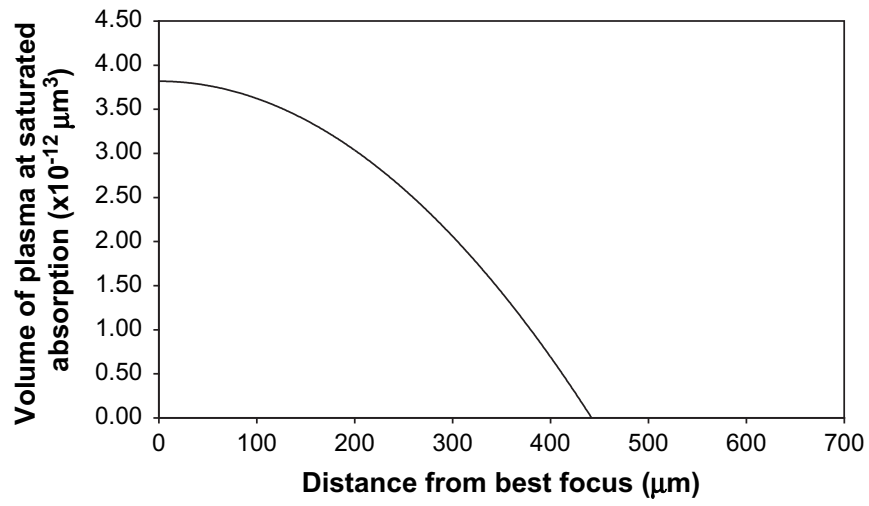

Fig. 4. Variation in volume at saturated absorption as a function of distance from focus according to a simple model described in the text. 

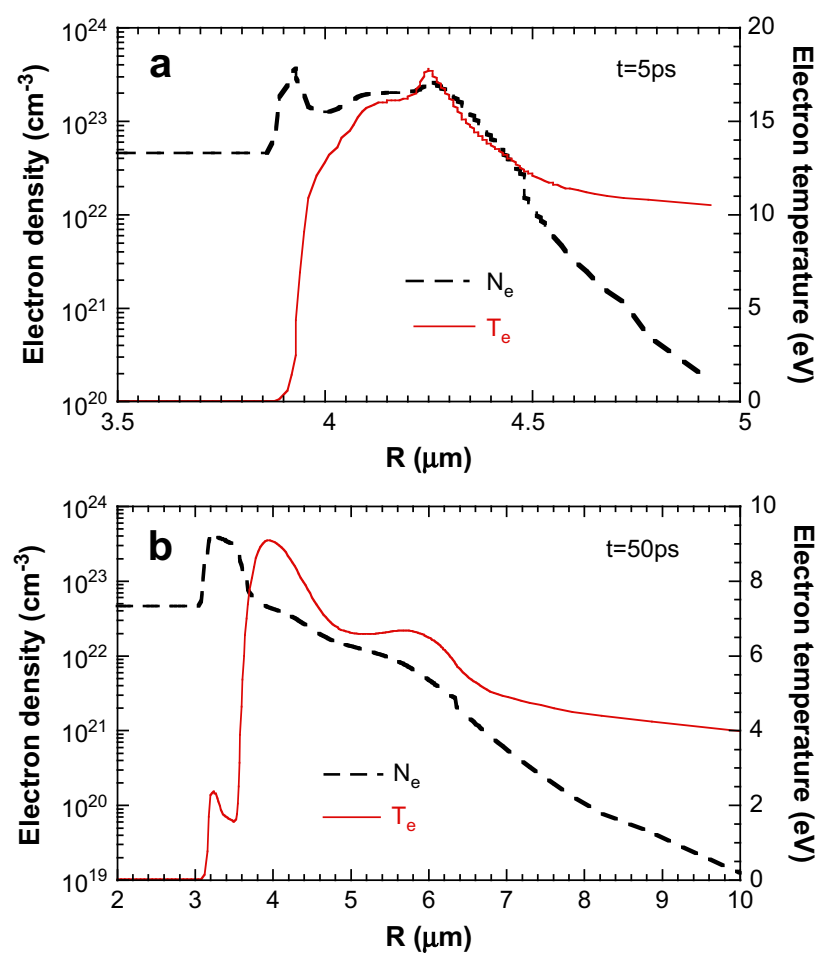

Fig. 5. Hyades simulations of the plasma evolution.

lines from O III to O VI present between $\sim 20 \mathrm{~nm}$ and $30 \mathrm{~nm}$ as identified from spectral databases [7].

The width of the $16.01 \mathrm{~nm}$ and $16.17 \mathrm{~nm}$ lines has been modelled and found to correspond to an electron density of $\sim 3.0 \pm 0.5 \times 10^{21} \mathrm{~cm}^{-3}$. This is somewhat lower than solid density but given the time integrated nature of the data is not unexpected. The temperature of the plasma has been estimated from the ratio of the $16 \mathrm{~nm}$ and $13 \mathrm{~nm}$ features, using both the line peaks and the integrated intensity under the lines and is shown in Fig. 3 as a function of focal position. The fact that the ratios are similar for both peak ratios and integrated ratios tends to indicate that we are in a low opacity regime, since the lines have oscillator strengths that are a factor of five different. As we can see, the inferred temperature does not vary very much with focal position (best focus on this scale is at $\mathrm{z}=300 \mu \mathrm{m}$ ). We believe that this is connected to the fact that in a parallel experiment to measure transmission of $53 \mathrm{~nm} \mathrm{Al}$ foils, as a function of intensity at $13.5 \mathrm{~nm}$, the absorption was seen to saturate at the highest intensity [10]. We do note that the intensity of the emission peaks at best focus. This we believe, is a result of the depth of the layer heated being thicker at higher intensity. The results of a simple model demonstrating this behaviour are shown in Fig. 4. We have assumed Gaussian optics with an $\mathrm{M}^{2}$ factor of 3 , based on study of the ablation craters in PMMA. The minimum spot diameter is set to $2.3 \mu \mathrm{m}$ and we calculate the volume of plasma for which the number of photons has reached one per absorbing atom. We can see that the volume decays over a distance of 200-300 $\mu \mathrm{m}$; broadly consistent with the data in Fig. 3a. It was noted in the data that the scattered FEL radiation decreased to a minimum at best focus for the converse reason, the surface area scattering is decreased and scatter from deeper within the target is reduced due to absorption on the way in and out of the target. The conclusions on the temperature at which the plasma lines are emitted are based on the assumption that the plasma lines are optically thin - we discuss this point further below.

\section{Simulation and discussion}

Modelling of transmission data [10] suggested that Al absorbs only 1 photon per atom and that most absorption should be within a few $\mathrm{nm}$ oxide layer at the surface and any hydrocarbon contaminants. In reference [11] it is shown that we should perhaps expect a layer of $5 \mathrm{~nm}$ contaminants on the target surface due to exposure to normal atmosphere. Accordingly, we model the plasma formation by using an energy dump in the HYADES hydro-dynamic code. We simulate an Al layer several microns thick with a top oxide layer of $10 \mathrm{~nm}$ and a $5 \mathrm{~nm}$ layer of $\mathrm{CH}$ to simulate hydrocarbon contamination. We have simulated the deposition of energy with a time dependent energy dump to the electrons that follows a $15 \mathrm{fs}$ FWHM pulse. In Fig. 5 we can see the resultant profiles of electron density and temperature at 5 ps and 50 ps. We can see that by 5 ps the plasma outer layers have cooled from $\sim 23 \mathrm{eV}$ peak temperature to $\sim 10 \mathrm{eV}$, just at the upper limit of the inferred temperatures from the spectra. Looking at the 50 ps case, it is clear that the plasma emission from Al IV can last many $10 \mathrm{~s}$ of picoseconds. Given the fact that the reciprocal of the A-rates of the transitions is question is $\sim 100 \mathrm{ps}$, we can make the assumption that the emission comes from the later time stages when the temperatures are indeed generally less than $10 \mathrm{eV}$. The lack of $\mathrm{Al} \mathrm{V}$ emission is consistent with the modest temperature reached. For the linear coefficient of $\mathrm{Al}$ the temperature would have reached $0.15 \mathrm{keV}$.

Interestingly, if we look at the continuum emission from $\mathrm{Al}$ and higher $\mathrm{Z}$ targets such as $\mathrm{Fe}$ and $\mathrm{Co}$, we see evidence of higher temperatures. In Fig. 6 we show emission from $\mathrm{Al}$ and both an $\mathrm{Fe}$ and a Co target, where we have an Al filter $(53 \mathrm{~nm})$ placed in front of the spectrometer to reduce stray light background. The L-edge of the Al can be seen in the data. In all cases we have made a fit to the continuum (excluding regions close to the scattered FEL in 1st and 2nd order and away from the Al IV lines). In order to do this, we

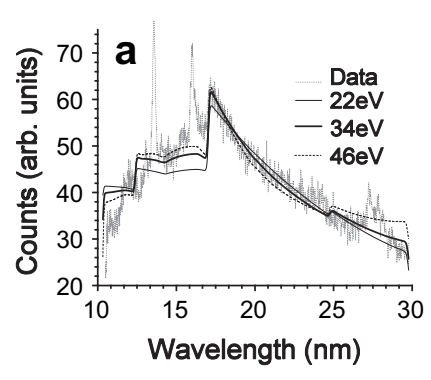

Al

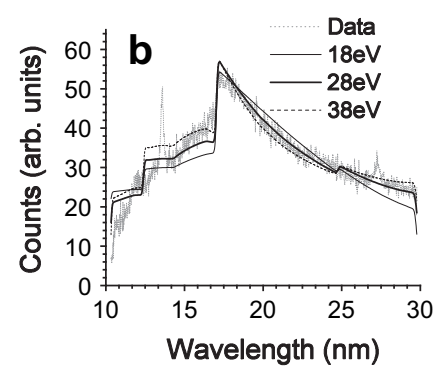

$\mathrm{Fe}$

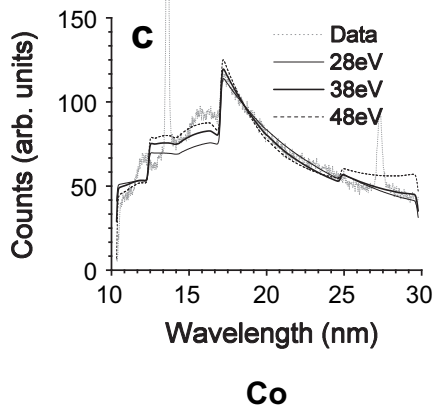

Co

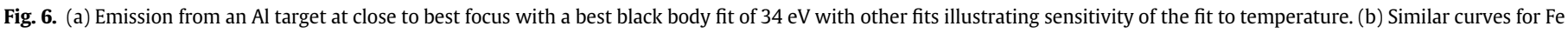
showing broadly similar fit temperature, (c) Similar curves for Co. 
have assumed that the absorbed FEL energy is rapidly thermalised at solid or near-solid density to form a dense plasma that is optically thick in the wavelength regime detected and emits as a black body.

The simulated curve is fitted to the data by a least squares fit with three variables; the temperature, a normalisation constant to scale the data to the simulation and a uniform background level to account for stray light and thermal noise in the CCD. We account for first, second and third order contributions, for which we know the relevant grating efficiencies [6], CCD detective quantum efficiency [12] and filter transmission [13]. The ratio of the FEL scatter in first and second order matches the grating calibration used. As can be seen, in all cases we get broadly similar temperatures of around 30$40 \mathrm{eV}$, which is a little higher than the temperatures seen in the $\mathrm{Al}$ simulation but still significantly lower than the expected temperatures for linear absorption. Simulations of the interaction with $\mathrm{Fe}$ indicate that with a linear absorption length of about $20 \mathrm{~nm}$ at this wavelength we would expect 68 photons per atom at the fluence used in the experiment and a temperature in excess of $0.1 \mathrm{keV}$. Again, it seems the evidence is consistent with the idea that the pulse is too fast for efficient absorption and saturation occurs.

In conclusion, emission from FEL irradiated solids has been studied for a variety of sample materials. Plasma emission lines from Al IV suggest temperatures of $5-8 \mathrm{eV}$ for irradiances of $10^{16} \mathrm{~W} \mathrm{~cm}^{-2}$ if we assume an optically thin LTE model, whilst emission line-widths suggest average electron densities of order a few times $10^{21} \mathrm{~cm}^{-3}$. This combined with the A-rates of the emission lines suggests that the Al IV emission is characteristic of late time in the expansion of the solid and that initial temperature is higher. However, if we consider, the absence of $\mathrm{Al} \mathrm{V}$ emission lines we can suggest that the peak temperature is not over $40 \mathrm{eV}$ this is in contrast to the expected heating of $\sim 0.15 \mathrm{keV}$ for the linear absorption coefficient of $\mathrm{Al}$ at this wavelength. The constancy of the temperature inferred over a wide range of focal positions is consistent with saturation of absorption and the increase of emission close to best focus can be explained by a simple model assuming the saturation. The observation of continuum emission from higher $Z$ targets can be made to fit a black-body emission curve if we assume a temperature of 30 to $40 \mathrm{eV}$. This may be characteristic of the temperatures initially created after the heated solid has equilibrated to a dense plasma.

\section{References}

[1] R.W. Lee, et al., JOSA B 20 (2003) 770.

[2] M. Fajardo, et al., European Physical Journal D 29 (2004) 69

[3] F.B. Rosmej, et al., J. Phys.: Conf. Ser. 72 (2007) 012007.

[4] R.W. Lee, et al., Laser and Particle Beams 20 (2002) 527.

[5] S. Bajt, et al., Proc. SPIE 7361, 18 (2009).

[6] D. Neely, Private communication.

[7] Y. Ralchenko, A.E. Kramida, J. Reader, et al., Atomic Spectra Database Website, http://physics.nist.gov/asd3.

[8] G. Tachiev, C. Froese Fischer URL, http://www.vuse.vanderbilt.edu/ cff/mchf collection/.

[9] V. Kaufman, M.C. Artru, W.L. Brillet, Journal of the Optical Society of America 64 (1974) 197.

[10] B. Nagler et al., Nature Physics, in press.

[11] R. Robinson available online at, http://volta.byu.edu/xray.html (2003).

[12] URL www.andor-tech.com.

[13] B.L. Henke, E.M. Gullikson, J.C. Davis, Atomic Data and Nuclear Data Tables 54 (1990) 181. 